\title{
NEOPLASIA VASCULAR DE BAIXO GRAU HEMANGIOPERICITOMA- RELATO DE CASO
}

\section{ARTIGO ORIGINAL}

PELIZER, Carlos Antônio Albuquerque ${ }^{1}$

ESPÓSITO, Mario Pinheiro ${ }^{2}$

OLIVEIRA, Ronan Djavier Alves ${ }^{3}$

PASSOS, Fabio Manoel dos ${ }^{4}$

PLACHESKI, Ana Carolina Galindo ${ }^{5}$

NETO, Alonso Alves Pereira ${ }^{6}$

ESPÓSITO, Guilherme Soriano Pinheiro ${ }^{7}$

\footnotetext{
${ }^{1}$ Residente em Otorrinolaringologia e Cirurgia Cérvico-Facial; Graduado em Medicina pelo Centro Universitário São Lucas - UNISL.

${ }^{2}$ Doutor em Otorrinolaringologia pela Faculdade de Ciências Médicas da Santa Casa de São Paulo.
}

${ }^{3}$ Residente em Otorrinolaringologia e Cirurgia Cérvico-Facial, Graduado em Medicina pela Universidade de Cuiabá - UNIC.

${ }^{4}$ Residente em Otorrinolaringologia e Cirurgia Cérvico-Facial; Graduado em Medicina pela Universidade Federal do Paraná - UFPR.

${ }^{5}$ Residente em Otorrinolaringologia e Cirurgia Cérvico-Facial; Graduada em Medicina pela Universidade de Cuiabá - UNIC.

${ }^{6}$ Residente em Otorrinolaringologia e Cirurgia Cérvico-Facial, Graduado em Medicina pela União das Faculdades dos Grandes Lagos - UNILAGO.

${ }^{7}$ Residente em Otorrinolaringologia e Cirurgia Cérvico-Facial; Graduado em Medicina pelo Centro Universitário São Lucas - UNISL. 
PELIZER, Carlos Antônio Albuquerque. Et al. Neoplasia vascular de baixo grau hemangiopericitoma- Relato de caso. Revista Científica Multidisciplinar Núcleo do Conhecimento. Ano 05, Ed. 08, Vol. 02, pp. 107-112. Agosto de 2020. ISSN: 24480959, Link de acesso: https://www.nucleodoconhecimento.com.br/saude/neoplasiavascular

\section{RESUMO}

O presente estudo propõe relatar o caso de uma paciente que fora diagnosticada com hemangiopericitoma, no Hospital de Otorrino, e como foi sucedido a realização do tratamento, perpassando por exames de anamnese completa, exame físico da orofaringe, cavidade nasal e ouvidos. O objetivo central é evidenciar o sucesso adquirido por meio da paciente através do modelo de tratamento proposto, sendo realizado cirúrgica excecional e biopsia com diagnóstico de neoplasia vascular de baixo grau, sendo assim, essa publicação tem por objetivo fomentar os estudos que reflitam na discussão deste tipo de tumor e relatar novas possibilidades de crescimento e locais de acometimentos.

Palavras-chave: Hemangiopericitoma, células vasculares.

\section{INTRODUÇÃO}

O hemangiopericitoma é um tumor de células vasculares (pericitos de Zimmerman) que se dispõem entre os capilares e vênulas da musculatura lisa. Sua primeira descrição diante da literatura se deu 1942, por meio da pesquisa feita por Stout e Murray[8], que descrevia-os como um tumor amorfo, ou seja, sem características macroscópicas que pudessem caracteriza-lo ou classifica-lo. O tumor representa apenas $1 \%$ de todos os tumores vasculares, como consta na literatura[9]. É um tumor de etiologia desconhecida e predileção por tronco e membros inferiores em sua maioria, sendo raros os casos em cabeça e pescoço, Stout e Murray (1942) lançaram na literatura 691 casos, mostrando que apenas nove destes eram hemangiopericitoma. Este tumor não possui quadro clínico ou apresentação em que 
se possa diagnosticar, sendo o diagnóstico somente possível através de imunohistoquímica.

\section{OBJETIVO}

Este trabalho visa relatar o caso de um paciente, no Hospital de Otorrino em Cuiabá - Mato Grosso, com diagnóstico de hemangiopericitoma em base de língua, bem como o tratamento de escolha realizado no hospital.

\section{METODOLOGIA}

Trabalho aprovado pelo Comitê de ética do Hospital Otorrino de Cuiabá - MT. De acordo com o estudo realizado por Nardi; Vieira; Pfuetzenreiter e Dedivitis, em sua pesquisa, Hemangiopericitoma de língua, afirma que as "Características angiográficas podem ajudar na diferenciação entre hemangiopericitomas e outros tipos de tumores hipervascularizados." (2011, n.p.), deste modo, se decidiu que o paciente em estudo fosse submetido a: anamnese completa, exame físico da orofaringe, cavidade nasal e ouvidos. Foi realizada revisão da literatura sobre a hemangiopericitoma acerca dos dados: idade, sexo, etiologia, sinais e sintomas, bem como critérios de diagnósticos e sobre tratamento de escolha.

\section{RELATO DE CASO}

Paciente G. F. dos S. de 44 anos, procura atendimento especializado em nosso serviço em Cuiabá - MT, em julho de 2019, devido a nodulação em base de língua à esquerda (tempo de início indefinido). A paciente nega sintomas associados, nega trauma local ou qualquer patologia prévia, desse modo, foi realizado uma videolaringofibroscopia para melhor elucidação e, também, para descartar quaisquer nódulos em outras regiões da laringe.

O tratamento proposto de imediato foi a exérese da lesão juntamente com anatomopatológico a ser realizado, posteriormente, foi realizado, em setembro de 2019, sem intercorrências e apresentando um pós-operatório satisfatório e sem 
recidiva da lesão. Seu estudo imuno-histoquímico apresentou neoplasia vascular de baixo grau sugestivo de hemangiopericitoma.

Figura 1: tumor na língua

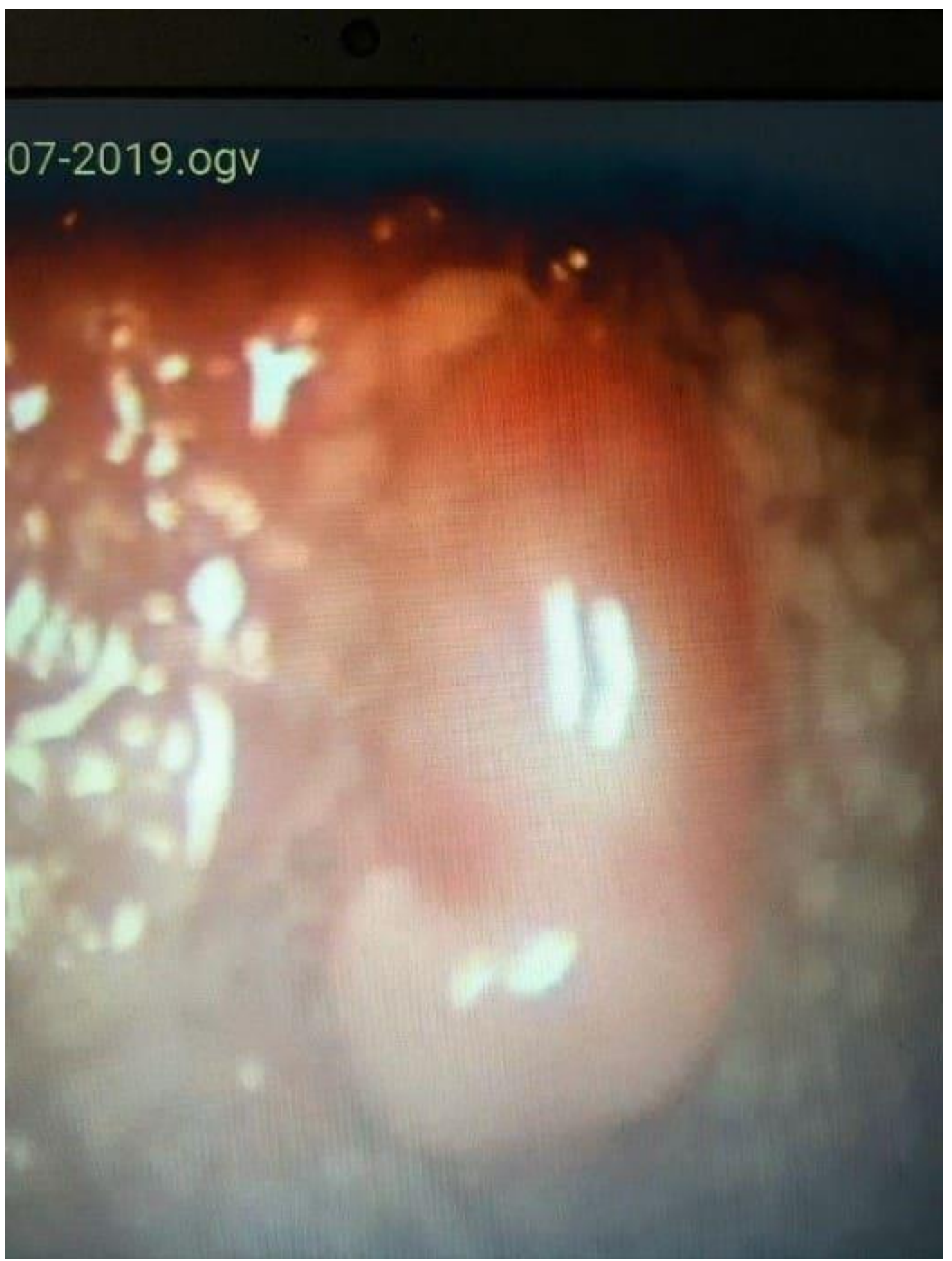

Fonte: Carlos Antônio Albuquerque Pelizer (2019) 


\section{DISCUSSÃO}

O hemangiopericitoma é um tumor raro, de crescimento lento e macroscopia irregular, sem qualquer característica que possa distingui-lo de outro tumor, ele é um tumor de células vasculares, mais propriamente dito dos pericitos de Zimmerman, que acompanham os capilares e vênulas no corpo, porém, possuem predileção em tronco e membros inferiores, o que os tornam difíceis de serem encontrados em cabeça e pescoço, ocorrendo em menos de $30 \%$ dos casos, como é descrito na pesquisa de Prado; Romano; Voegels e Butang (2004). O tumor não possui preferência por gênero e seu acometimento está mais descrito em adultos. Por não apresentar característica própria seu diagnóstico macroscópico fica impossibilitado, sendo possível somente realizar através de imuno-histoquímica, em que irá identificar células reativas aos marcadores CD34, actina e fator VIII.

De acordo com a Caparroz e Dedivitis (2013), o melhor tratamento a ser proposto devia ser a exérese total da lesão para evitar aumento da lesão e também metástase, além de recidiva. Além do mais, é necessário que se fizesse exames de imagem como: ressonância magnética, para que houvesse uma avaliação de infiltração de tumor para cavidades, sendo assim obrigatórias, portanto, para a paciente do caso relatado foi realizado a exérese da lesão e acompanhamento ambulatorial para avaliação de recidiva.

\section{CONCLUSÃO}

As neoplasias caracterizadas como hemangiopericitoma são raras de identificar, porém o profissional deve estar atento a qualquer lesão ou nodulação de cavidade oral. Deve-se solicitar exames de imagem para avaliação de infiltração sempre e, também, uma biopsia para identificar com o que irá se deparar no momento, somente assim poderá traçar a melhor conduta. Em geral, é indicado para este tumor a cirurgia. 


\section{REFERÊNCIAS}

ABRAHÃO, Márcio; CARVALHO, Jomar Rezende; CERVANTES, Onivaldo; FILHO, Marcílio Ferreira Marques; HADDAD, Leonardo; LEONHARDT, Fernando Danelon; SANTOS, Rodrigo de Oliveira. Hemangiopericitoma maligno de cabeça e pescoço em uma criança: relato de caso. Revista: São Paulo Medical Journal. São Paulo, vol.122, ㄲo5, 2004. Disponível em: <http://www.scielo.br/scielo.php?pid=S151631802004000500010\&script=sci_arttext\&tIng=pt> Acesso em: 11 de fevereiro de 2020.

BEZZERRA, Kelen Chistina A.; REIS, Djulienne Marly K.; PRICHOA, Franciele Carla. Tumor glômico de parede abdominal. Santa Catarina, 2019. Disponível em: $<$ http://www.scielo.br/pdf/jbpml/v55n1/pt_1676-2444-jbpml-55-01-0114.pdf> Acesso em: 11 de fevereiro de 2020.

BOYADZHIEV, Georgi; BRADLEY, Patrick; NIKIFOROVA, Lora; SAPUNDZHIEV, Nikolay. Fulminant hemangiopericytoma of the larynx - a case report and a review of the literature. Brazilian Journal of Otorhinolaryngology, 2017. Disponível em: <http://www.bjorl.org.br/pt-pdf-S2530053917302389> Acesso em: 11 de fevereiro de 2020 .

CAPARROZ, Fábio de Azevedo; CERVANTES, Onivaldo; GREGGIO, Barbara; PALUMBO, Marcel das Neves; WALDER, Fernando. Hemangiopericitoma de valécula em paciente jovem do sexo masculino: relato de caso. Revista: Brasileira Cir. Cabeça e Pescoço, vol. 42, n¹, pág. 41 - 43, 2013. Disponível em: $<$ https://www.sbccp.org.br/wp-content/uploads/2014/11/REVISTA-SBCCP-42-1artigo-09.pdf > Acesso em: 11 de fevereiro de 2020.

DEDIVITIS, Rogério Aparecido; NARDI, Carlos Eduardo Molinari; PFUETZENREITER, Elio Gilberto; VIEIRA, Victor Bandini. Hemangiopericitoma de língua. Revista: Brazilian Jornal of Otorhinolaryngology. São Paulo, vol.78 ํo.2, 2012. Disponível em: <http://www.scielo.br/scielo.php?script=sci_arttext\&pid=S180886942012000200024> Acesso em: 11 de fevereiro de 2020. 
Hemagiopericytoma. Rare diseases, 2013. Disponível em: $<$ https://rarediseases.info.nih.gov/diseases/2627/hemangiopericytoma> Acesso em: 11 de fevereiro de 2020.

KONNO, Sérgio Nobuo. Neoplasias da Cavidade oral e da orofaringe. 2005. Disponível em: <https://forl.org.br/Content/pdf/seminarios/seminario_57.pdf> Acesso em: 11 de fevereiro de 2020.

MURRAY, Margaret R.; STOUT, Arthur Purdy. Hemangiopericytoma: A vascular tumor featuring zimmermann's pericytes. Annais of Surgery. Nova York, vol. 116, №1, $1942 . \quad$ Disponível em: $<$ https://www.ncbi.nlm.nih.gov/pmc/articles/PMC1543753/pdf/annsurg013390035.pdf> Acesso em: 11 de fevereiro de 2020.

PRADO FAP; ROMANO FR; VOEGELS RL; BUTUGAN O. Hemangiopericitoma de seio esfenoidal. Arq Otorrinolaringol. 2004. Disponível em: $<$ http://arquivosdeorl.org.br/additional/acervo_port.asp?id=288 > Acesso em: 23 de junho de 2020.

\section{APÊNDICE - REFERÊNCIAS DE NOTA DE RODAPÉ}

8. O texto citado é encontrado na base literária médica, que se encontra com o nome de "Hemangiopericytoma: Vascular tumor featuring Zimmermann's pericytes".

9. Tal informação é sobreposta no texto, "Hemangiopericitoma maligno de cabeça e pescoço em uma criança: relato de caso".

Enviado: Fevereiro, 2020.

Aprovado: Agosto, 2020. 